\title{
Engineering terpenoid production through transient expression in Nicotiana benthamiana
}

\author{
James Reed ${ }^{1} \cdot$ Anne Osbourn ${ }^{1}$ (1) \\ Received: 5 March 2018 / Accepted: 12 May 2018 / Published online: 21 May 2018 \\ (c) The Author(s) 2018
}

\begin{abstract}
Terpenoids are the most structurally diverse class of plant natural products with a huge range of commercial and medical applications. Exploiting this enormous potential has historically been hindered due to low levels of these compounds in their natural sources, making isolation difficult, while their structural complexity frequently makes synthetic chemistry approaches uneconomical. Engineering terpenoid biosynthesis in heterologous host production platforms provides a means to overcome these obstacles. In particular, plant-based production systems are attractive as they provide the compartmentalisation and cofactors necessary for the transfer of functional pathways from other plants. Nicotiana benthamiana, a wild relative of tobacco, has become increasingly popular as a heterologous expression platform for reconstituting plant natural product pathways, because it is amenable to Agrobacterium-mediated transient expression, a scalable and highly flexible process that enables rapid expression of genes and enzymes from other plant species. Here, we review recent work describing terpene production in $N$. benthamiana. We examine various strategies taken to engineer this host for increased production of the target metabolite. We also look at how transient expression can be utilised for rapid generation of molecular diversity, including new-to-nature products. Finally, we highlight current issues surrounding this expression platform and discuss the future directions and developments which will be needed to fully realise the potential of this system.
\end{abstract}

Keywords Metabolic engineering $\cdot$ Agroinfiltration $\cdot$ Molecular diversity $\cdot$ Combinatorial biosynthesis $\cdot$ Natural products · Synthetic biology

\section{Introduction}

Plant natural products have long provided humanity with a source of flavourings, fragrances, dyes, cosmetics, and medicines. The plant kingdom remains a major source of important pharmaceuticals including painkillers and anticancer agents (Atanasov et al. 2015). However, accessing sufficient quantities of these compounds from the producing species frequently proves challenging due to low abundance of the target metabolite, slow growth or difficulty in propagation of the plant of origin and the challenges of purifying compounds from chemically complex plant extracts. Furthermore, the structural complexity of many plant natural

Communicated by Neal Stewart.

Anne Osbourn

anne.osbourn@jic.ac.uk

1 Department of Metabolic Biology, John Innes Centre, Norwich Research Park, Norwich NR4 7UH, UK products makes current methods of chemical synthesis unfeasible or uneconomical. As our understanding of plant natural product biosynthesis increases, expression of these pathways in heterologous hosts provides an opportunity to address these problems to meet the continued need for both currently used and new natural products.

Nicotiana benthamiana, a wild relative of tobacco, has seen increasing use over the past decade as a system for reconstituting plant natural product biosynthetic pathways. There are two main reasons underlying this. First, as a higher plant, $N$. benthamiana shares common cellular compartmentalisation, cofactors and coenzymes with other plants, thereby enabling the transfer of pathways from other plants without the need to extensively optimise the system. Second, this plant species is highly amenable to Agrobacteriummediated transient expression, a process in which leaves are infiltrated using a needle-less syringe with a suspension of Agrobacterium tumefaciens carrying the gene(s) of interest within a binary vector; a process referred to as agroinfiltration (Fig. 1a). This approach is rapid, with gene expression 
(a)

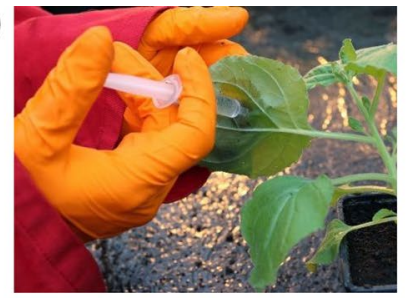

(b)

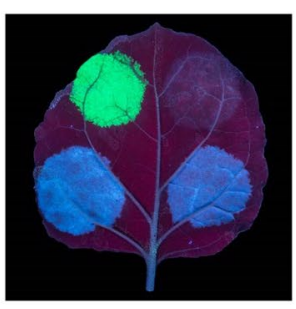

(c)
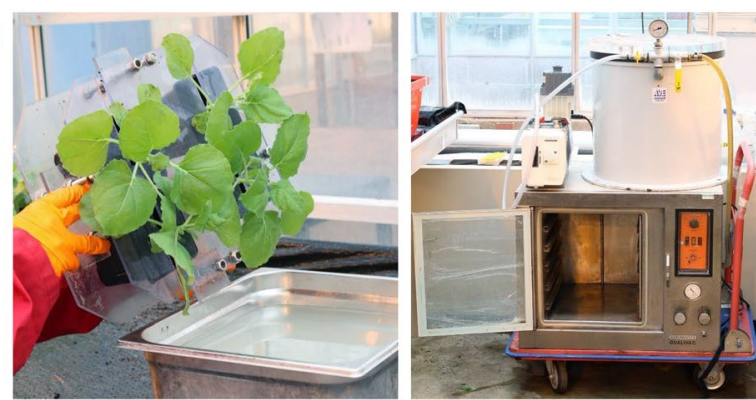

(d)

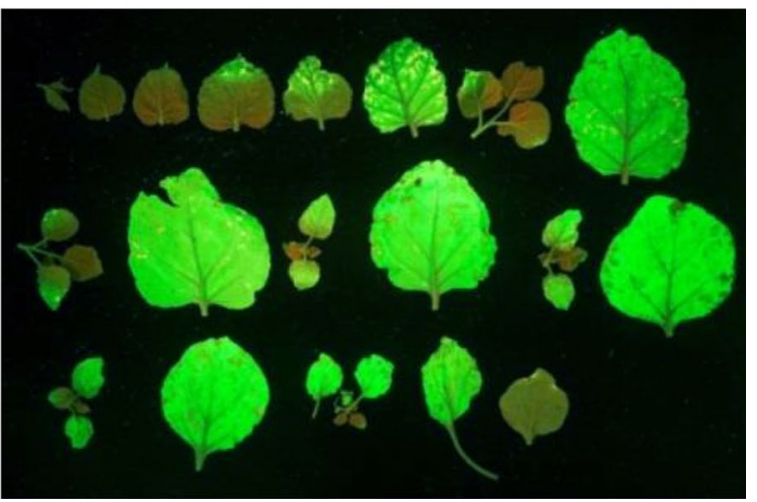

Fig. 1 Transient expression in N. benthamiana. a Hand infiltration of $N$. benthamiana with a suspension of $A$. tumefaciens using a needle-less syringe. $\mathbf{b}$ Transient expression of protein and metabolites in $N$. benthamiana. Leaf viewed under UV 5 days after infiltration. Upper left-expression of green fluorescent protein. Bottom left and right-production of UV-fluorescent $N$-methyl anthranilate through expression of an oat methyltransferase and glycosyltransferase. Upper right-empty vector control. Picture courtesy of Aymeric Leveau and the Norwich Research Park (NRP) Image Library (c) Vacuum infiltration of $N$. benthamiana. Plants are inverted into a suspension of A. tumefaciens (left) and placed into a vacuum chamber (right). The vacuum is used to draw air from the interstitial leaf space, which is replaced by the inoculum upon release of the vacuum. $\mathbf{d}$ Leaves from a single $N$. benthamiana plant 5 days after vacuum infiltration with a GFP-carrying A. tumefaciens. Infiltration of most of the aerial parts of the plant is possible using this method. Leaves are arranged from top left to bottom right based on their height order (top-bottom) on the plant. This method provides much better coverage compared to infiltration by hand. Figure adapted from Reed et al. (2017)

and detection of the target protein or product typically achieved within a few days (Fig. 1b). This property also makes $N$. benthamiana a popular host for the production of proteins such as virus-like particles (VLPs), which have significant utility as vaccines (Marsian and Lomonossoff 2016). Agroinfiltration is a highly flexible process; expression of multiple genes can easily be achieved by simultaneous coinfiltration of A. tumefaciens strains, each containing different expression constructs. This approach thereby enables expression of multi-step pathways or combinatorial biosynthetic libraries, without the need to build multi-gene constructs (discussed further below). While the necessary metabolic precursors are often present in sufficient quantities in $N$. benthamiana for the heterologously expressed enzymes to function, it is also possible to infiltrate such precursors into the leaves (Lau and Sattely 2015; Miettinen et al. 2014). This may be useful for testing enzyme activity towards specific substrates, or where the necessary precursors cannot be produced in sufficient quantities $N$. benthamiana. Finally, agroinfiltration can be scaled to infiltrate large numbers of plants through vacuum infiltration (Fig. 1c/d).

So far, pathways for several different types of natural products have been successfully reconstituted in N. benthamiana, including for alkaloids (Miettinen et al. 2014), lignans (Lau and Sattely 2015), cyanogens (Rajniak et al. 2015), betalains (Polturak et al. 2016), ketides (AndersenRanberg et al. 2017) and glucosinolates (Crocoll et al. 2016; Geu-Flores et al. 2009; Pfalz et al. 2011). These successes have demonstrated the potential of $N$. benthamiana for production of plant specialised metabolites. This review aims to highlight some key examples, focusing on terpene production. Terpenes (or isoprenoids) are the most structurally diverse class of plant specialised metabolites. They have a long historical use as flavourings and fragrances, but there are also numerous examples of high-value terpene therapeutics, such as the anti-cancer diterpene taxol, the anti-malarial sesquiterpene artemisinin and the triterpene vaccine adjuvant QS-21 (Fig. 2a). The difficulty of obtaining these compounds from their natural sources has driven the search for a sustainable and affordable supply. This in turn has prompted a large number of studies looking to engineer production of these and other compounds in heterologous hosts.

\section{Engineering terpene production in $\mathbf{N}$. benthamiana}

Most eukaryotes, including animals, fungi and higher plants share a common pathway for terpenoid biosynthesis, known as the mevalonate (MVA) pathway. However, in addition to the MVA pathway, plants possess a second, plastidial 2-C-methyl-D-erythritol 4-phosphate (MEP) pathway (Lichtenthaler et al. 1997). Both pathways produce common 5-carbon building blocks isopentenyl diphosphate (IPP) and its isomer dimethylallyl diphosphate (DMAPP), but are derived from different starting substrates (Fig. 2b). Although crosstalk between these pathways may occur (Bick and Lange 2003; Hemmerlin et al. 2003, 2012; Schuhr et al. 2003), the MEP pathway is generally considered responsible for production of 
(a)

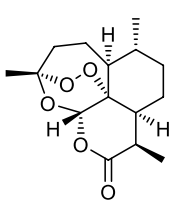

Artemisinin Sesquiterpene Anti-malarial

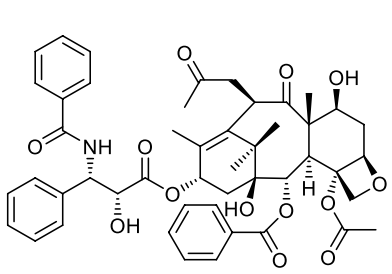

Taxol (Paclitaxel) Diterpene Antitumour agent

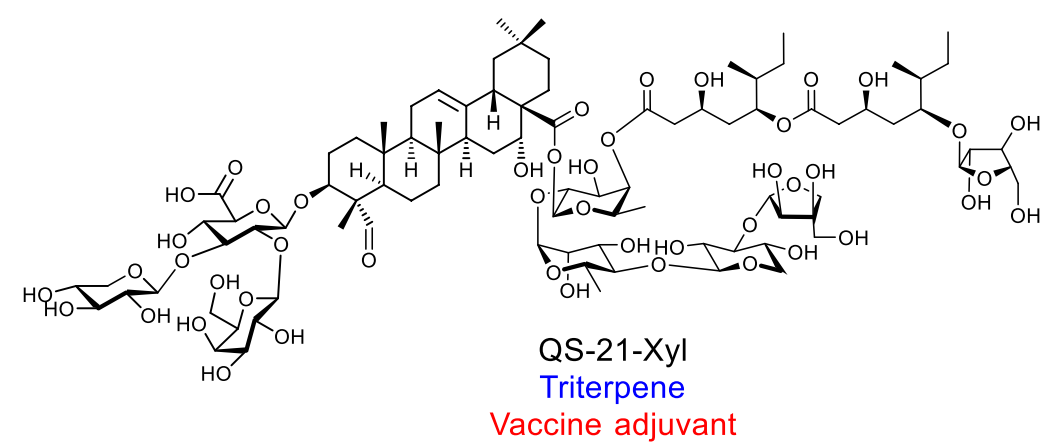

(b)

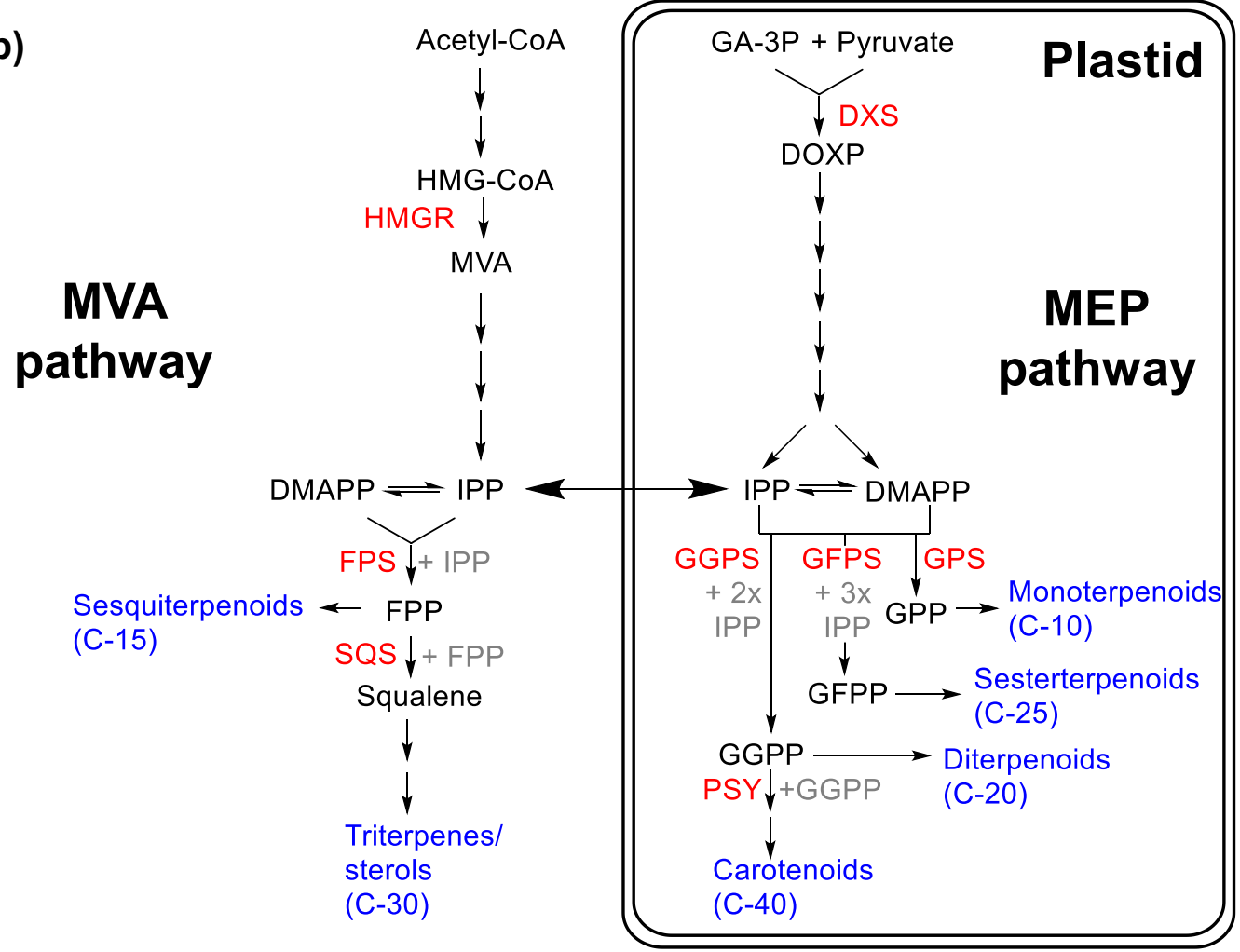

Fig. 2 Terpene production in plants. a Examples of some medically important terpenes produced by plants. The names and class of the products are given in black and red, respectively, with the medical applications in blue. b Overview of the two terpene biosynthetic pathways in plant cells. Key biochemical intermediates are named in black, with important enzymes described in the main text named in red. The various classes of end products are named in blue. The MVA pathway is localised to the cytosol and associated endomembranes and is responsible for the production of sesquiterpenes and triterpenes. The MEP pathway is localised to the plastid and synthesises

monoterpenes (C-10), diterpenes (C-20) and tetraterpenes/carotenoids (C-40), while sesquiterpenes (C-15) and triterpenes (C-30) are synthesised via the MVA pathway (Vranova et al. 2013). This situation is conserved across higher plants, thus terpene biosynthetic enzymes derived monoterpenes, diterpenes, sesterterpenes and carotenoids (tetraterpenes). CoA Coenzyme A; $H M G$ 3-hydroxy, 3-methylglutaryl; $M V A$ mevalonate; IPP isopentenyl diphosphate; DMAPP dimethylallyl diphosphate; FPP farnesyl diphosphate; DOXP 1-deoxy-D-xylulose 5-phosphate; GPP geranyl diphosphate; GFPP geranylfarnesyl diphosphate; GGPP geranylgeranyl diphosphate, HMGR HMG-CoA reductase; FPS FPP synthase; SQS Squalene synthase; DXS 1-deoxyD-xylulose 5-phosphate synthase; GPS geranyl-diphosphate synthase; GFPS geranylfarnesyl-diphosphate synthase; GGPS geranylgeranyldiphosphate synthase, $P S Y$ phytoene synthase

from distantly related plant species generally seem to be correctly targeted and function appropriately in N. benthamiana. For example, sesterterpenes are a rare class of plastid-derived C-25 terpenes that are not naturally produced in $N$. benthamiana. Until recently, the terpene synthases 
that make these compounds (sesterterpene synthases) had not been characterised from plants. However, such enzymes have recently been identified in Brassicaceae species. Coexpression of various $A$. thaliana geranylfarnesyl diphosphate synthases (GFPS) with sesterterpene synthases allowed for production of various cyclised sesterterpenes in N. benthamiana (Huang et al. 2017b). In addition, the yield of the target pathway end product can often be enhanced by overcoming upstream pathway bottlenecks or countering the flow of intermediates into competing pathways. Hence, a good understanding of the pathway relevant to the metabolite of interest is key to successful engineering of the intended metabolite. Thus, significant attention has been given to understanding the important regulatory enzymes in each of the two pathways, and how they influence yield. Transient expression, therefore, provides a rapid method of screening candidate genes.

For the MVA pathway, 3-hydroxy-3-methylglutarylCoA reductase (HMGR) is known to be a key rate-limiting enzyme. The N-terminus of HMGR has an important regulatory function but is dispensable for catalytic activity. Truncation of this region results in a feedback-insensitive protein (tHMGR) and enhances yields of MVA end products such as squalene (Polakowski et al. 1998). This approach forms a standard strategy for enhancing production of MVA-derived sesquiterpenes and triterpenes (Cankar et al. 2015; Liu et al. 2014; Reed et al. 2017; van Herpen et al. 2010) (Table 1; Fig. 2). For MEP-derived mono- and diterpenes, expression of a prenyltransferase enzyme producing the relevant $\mathrm{C}-10$ or C-20 prenyl diphosphate substrate can enhance yields

Table 1 Examples of various MVA-derived terpenes produced through transient expression in N. benthamiana

\begin{tabular}{|c|c|c|c|c|c|}
\hline Compound & Class & Quantity & Strategy & Fold increases & References \\
\hline Linalool/Caryophyllene & Mono/Sesquiterpene & - & $\begin{array}{l}\text { RNAi of } N \text {. benthami- } \\
\text { ana VAMP72 genes }\end{array}$ & $\begin{array}{l}\text { Fivefold increase upon } \\
\text { silencing VAMP72 } \\
\text { genes }\end{array}$ & Ting et al. (2015) \\
\hline Costunolide & Sesquiterpene & $60 \mathrm{ng} / \mathrm{g} \mathrm{FW}$ & $\begin{array}{l}\text { Targeting to mitochon- } \\
\text { dria }\end{array}$ & $\begin{array}{l}\text { 15-fold increases from } \\
\text { mitochondrial-target- } \\
\text { ing versus cytosol }\end{array}$ & Liu et al. (2011) \\
\hline Parthenolide & Sesquiterpene & $1.4 \mu \mathrm{g} / \mathrm{g} \mathrm{FW}$ & Expression of HMGR & $\begin{array}{l}\text { Fourfold increases } \\
\text { in the parthenolide } \\
\text { precursor costunolide } \\
\text { with HMGR }\end{array}$ & Liu et al. (2014) \\
\hline$(+)$-Valencene & Sesquiterpene & $\begin{array}{l}0.70 \mu \mathrm{g} / \mathrm{g} \mathrm{FW} / 24 \mathrm{~h} \\
\text { (unopti-mised) }\end{array}$ & $\begin{array}{l}\text { Expression of tHMGR } \\
\text { \& FPS, silencing of } \\
\text { SQS and EAS }\end{array}$ & $\begin{array}{l}\text { 2.9-fold increases } \\
\text { from expression of } \\
\text { tHMGR and FPS }\end{array}$ & Cankar et al. (2015) \\
\hline Artemisinic acid & Sesquiterpene & $16.6 \mathrm{mg} / \mathrm{kg} \mathrm{FW}$ & $\begin{array}{l}\text { Genes fused together } \\
\text { with use of } 2 \mathrm{~A} \\
\text { ribosomal skip- } \\
\text { ping sequences/ } \\
\text { mitochondrial- } \\
\text { targeting of ADS and } \\
\text { FPS/ expression of } \\
\text { tHMGR }\end{array}$ & $\begin{array}{l}\text { Use of the fusion } \\
\text { construct with mito- } \\
\text { chondrial FPS and } \\
\text { tHMGR increased } \\
\text { amorphadiene } \\
\text { (artemisinic acid pre- } \\
\text { cursor) in headspace } \\
\text { by } \sim \text { twofold, and } \\
\text { internal leaf amor- } \\
\text { phadiene by } \sim 7 \text {-fold }\end{array}$ & van Herpen et al. (2010) \\
\hline Artemisinin & Sesquiterpene & $3 \mathrm{ng} / \mathrm{mg} \mathrm{DW}$ & $\begin{array}{l}\text { Expression of LTP3 } \\
\text { and PD2 plus HMGR }\end{array}$ & $\begin{array}{l}\text { Approx } 50 \% \text { increase } \\
\text { in artemisinin at } 13 \\
\text { days after infiltration }\end{array}$ & Wang et al. (2016) \\
\hline $\begin{array}{l}\text { 12,13-epoxy, } \\
\text { 16-hydroxy- } \beta \text {-amyrin }\end{array}$ & Triterpene & $1.18 \mathrm{mg} / \mathrm{g} \mathrm{DW}$ & - & - & Geisler et al. (2013) \\
\hline Various & Triterpene & 0.12-3.3 mg/g DW & $\begin{array}{l}\text { tHMGR, vacuum } \\
\text { infiltration }\end{array}$ & $\begin{array}{l}\text { Fourfold increases } \\
\text { in } \beta \text {-amyrin upon } \\
\text { expression of } \\
\text { tHMGR }\end{array}$ & Reed et al. (2017) \\
\hline
\end{tabular}

An emphasis is placed on studies reporting either a specific yield or those which describe engineering approaches to improve yields of target compounds. Quantities are reported as given in the referenced study, these generally correspond to predicted yields from GC- or LC- quantification. Where isolated yields are reported, these are highlighted in bold. Where engineering strategies are described, the approximate fold increase in target compound is also given

$F W$ Fresh weight, $D W$ dry weight, VAMP vesicle-associated membrane protein, $(t) H M G R$ HMG-CoA reductase ("t" denotes N-terminal-truncated form), FPS farnesyl diphosphate synthase, ADS amorphadiene synthase, SQS squalene synthase, EAS 5-Epi-aristolochene synthase, LTP3 lipid transfer protein 3,PD2 pleiotropic drug resistance 2 
(Dong et al. 2016; Yin et al. 2017). However, it may also be necessary to heterologously express the first committed MEP pathway enzyme, 1-deoxy-D-xylulose 5-phosphate synthase (DXS) to enhance the accumulation of end products (Andersen-Ranberg et al. 2016; Brückner and Tissier 2013) (Table 2; Fig. 2). The choice of plant species from which the yield-boosting enzymes are derived does not seem to be critical, for example the above studies utilised HMGR enzymes derived from oat or A. thaliana (Cankar et al. 2015; Liu et al. 2014; Reed et al. 2017; van Herpen et al. 2010), DXS from Solanum lycopersicum or Coleus forskohlii and prenyl transferases from peppermint, C. forskohlii and Nicotiana tabacum. At the same time, differences in relative activity can influence the extent to which an enzyme may be effective at boosting yields and testing multiple isoforms may be necessary (Dong et al. 2016).

Overexpression of these key enzymes is usually sufficient to enhance accumulation of the target compounds severalfold (Tables 1, 2). Nevertheless, expression or silencing of other enzymes at key branch points in the pathway has also been employed as a strategy to further enhance yields.
For example, in the MVA pathway, a 2.8-fold increase in sesquiterpene $(+)$-valencene levels was achieved by simultaneous silencing of endogenous $N$. benthamiana squalene synthase (SQS) and sesquiterpene (5-epi-aristolochene) synthase which compete with the heterologous valencene synthase for available substrate (farnesyl diphosphate, FPP) (Cankar et al. 2015). SQS appears to be limiting for triterpene production, since transient expression of an oat SQS has been shown to enhance yields of $\beta$-amyrin by 2-3-fold (Reed et al. 2017). For the MEP pathway, Hasan et al. (2014) demonstrated that yields of taxadiene (the core scaffold for the diterpene anti-cancer agent taxol), could be enhanced in $N$. benthamiana through silencing of endogenous phytoene synthase (PSY), which competes with taxadiene synthase for geranylgeranyl diphosphate (GGPP). This approach was sufficient to enhance taxadiene levels almost twofold from $25 \mu \mathrm{g} / \mathrm{g}$ dry leaf weight (DW) in control lines to $48 \mu \mathrm{g} / \mathrm{g}$ DW (Hasan et al. 2014). Finally, improved yield of the target compound can also be achieved by targeting processes outside of the immediate biosynthetic pathway. For example, one interesting study employed agroinfiltration to silence

Table 2 Examples of various MEP-derived terpenes produced through transient expression in $N$. benthamiana

\begin{tabular}{|c|c|c|c|c|c|}
\hline Compound & Class & Quantity & Strategy & Fold increases & References \\
\hline Various & Monoterpene & $5.55-19.08 \mu \mathrm{g} / \mathrm{g}$ FW/24 h & $\begin{array}{l}\text { Expression of GPPS } \\
\text { small subunit }\end{array}$ & $\begin{array}{l}\sim 4-5 \text { fold increases of } \\
(-) \text { linalool }\end{array}$ & Yin et al. (2017) \\
\hline Geraniol & Monoterpene & $27 \mu \mathrm{g} / \mathrm{g} \mathrm{FW}$ & - & - & Vasilev et al. (2014) \\
\hline Geraniol & Monoterpene & $93 \mu \mathrm{g} / \mathrm{g}$ FW & - & - & Fischer et al. (2013) \\
\hline Geraniol & Monoterpene & $129 \mu \mathrm{g} / \mathrm{g} \mathrm{FW}$ & $\begin{array}{l}\text { Targeting to different sub- } \\
\text { cellular compartments } \\
\text { (cytosol, mitochondria } \\
\text { and plastid) }\end{array}$ & $\begin{array}{l}\text { 2-3-fold increases } \\
\text { with plastid-targeted } \\
\text { GES and GPPS versus } \\
\text { plastid-targeted GES } \\
\text { alone }\end{array}$ & Dong et al. (2016) \\
\hline $\begin{array}{l}\text { 18-Hydroxy } \\
\text { dolabella- } \\
\text { 3,7-diene }\end{array}$ & Diterpene & $0.26 \mathrm{mg} / \mathrm{g} \mathrm{FW}$ & Targeting to mitochondria & - & Dickschat et al. (2017) \\
\hline Isopimaric acid & Diterpene & $45-55 \mu \mathrm{g} / \mathrm{g}$ DW & $\begin{array}{l}\text { Expression of DXS and } \\
\text { GGPPS }\end{array}$ & 3 -fold & Gnanasekaran et al. (2015) \\
\hline Taxadiene & Diterpene & $48 \mu \mathrm{g} / \mathrm{g}$ DW & Silencing of native PSY & 1.9-fold & Hasan et al. (2014) \\
\hline Various & Diterpene & $0.5-5 \mathrm{mg}$ & $\begin{array}{l}\text { Expression of DXS and } \\
\text { GGPPS vacuum infiltra- } \\
\text { tion }\end{array}$ & $\begin{array}{l}\sim 10 \text {-fold increases with } \\
\text { combination of DXS } \\
\text { and GGPPS }\end{array}$ & $\begin{array}{l}\text { Andersen-Ranberg et al. } \\
\text { (2016) }\end{array}$ \\
\hline Cembratrienol & Diterpene & $2500 \mathrm{ng} / \mathrm{cm}^{2}$ & $\begin{array}{l}\text { Coexpression of DXS and } \\
\text { GGPPS }\end{array}$ & $\begin{array}{l}\text { 3.5-fold increase with } \\
\text { DXS + GGPPS } 2\end{array}$ & $\begin{array}{l}\text { Brückner and Tissier } \\
\text { (2013) }\end{array}$ \\
\hline Various & Sesterterpene & $0.03-1.13 \mathrm{mg} / \mathrm{g}$ DW & Vacuum infiltration & - & Huang et al. (2017b) \\
\hline Various & Sesterterpene & $0.02-0.97 \mathrm{mg} / \mathrm{g}$ DW & $\begin{array}{l}\text { Expression of DXS, } \\
\text { vacuum infiltration }\end{array}$ & - & Huang et al. (2017a) \\
\hline
\end{tabular}

An emphasis is placed on studies reporting either a specific yield or those which describe engineering approaches to improve yields of target compounds. Quantities are reported as given in the referenced study, these generally correspond to predicted yields from GC- or LC- quantification. Where isolated yields are reported, these are highlighted in bold. Where engineering strategies are described, the approximate fold increase in target compound is also given

$F W$ Fresh weight, $D W$ dry weight, GPPS geranyl-diphosphate synthase, GGPPS geranylgeranyl-diphosphate synthase, GES geraniol synthase, $D X S$ 1-deoxy-D-xylulose 5-phosphate synthase, PSY phytoene synthase

${ }^{\text {a }}$ This is a bacterial diterpene synthase derived from Chitinophaga pinensis 
$N$. benthamiana SNARE proteins (which mediate vesicular fusion) with simultaneous transient expression of linalool and caryophyllene synthases. This approach increased the levels of products by fivefold relative to non-silenced controls (Ting et al. 2015). Surprisingly this appeared to be an indirect effect, and was linked to reduced proteasome function, concordant with increased stability of the ectopically expressed terpene synthase proteins.

Other approaches to engineering terpene production in $N$. benthamiana have aimed to exploit the compartmentalisation of isoprenoid biosynthetic pathways in plants. By addition (or removal) of target peptides, the subcellular location of the transiently expressed protein can be altered in $N$. benthamiana, thereby deriving substrates from a different source to the native protein. For example, targeting sesquiterpene synthases to the mitochondria has been explored in several studies (Eljounaidi et al. 2014; Liu et al. 2011; van Herpen et al. 2010). In one case, this approach resulted in 15 -fold higher accumulation of the target metabolite (costunolide) versus the native, cytosolic protein (Liu et al. 2011), suggesting that FPP may be more readily available in this compartment. Mitochondria do not appear to have a dedicated terpenoid biosynthesis pathway, relying on import of IPP from other compartments (Disch et al. 1998); therefore, the apparent reason behind the success of this strategy is poorly understood. In another example, transient expression was used to probe the capacity of chloroplasts, mitochondria and cytosol for biosynthesis of the monoterpene geraniol by systematic targeting of a geraniol synthase (GES) and geranyl-diphosphate synthase (GPS) to each compartment (Dong et al. 2016). This revealed that targeting to the plastids (the native compartment for GPS and GES) resulted in the highest levels of geraniol and derivatives, followed by mitochondrial and cytosolic targeting. Interestingly, in this study replacing the native GES plastid targeting peptide with an artificial version resulted in a moderate (1.2-fold) increase in geraniol (Dong et al. 2016). The results of this and similar studies clearly demonstrate that transient expression is an excellent means to rapidly probe various biosynthetic strategies for optimising target yields. Altering the subcellular compartments of terpene synthases has also been shown to be a valid strategy for increasing yields in transgenic plants (Farhi et al. 2011; Wu et al. 2006, 2012). Therefore, the findings of these studies may be used to inform strategies for regulating expression in stable lines.

\section{Rapid engineering of molecular diversity through transient expression}

Enzymes in specialised metabolism often display activity towards non-native substrates, a phenomenon which can be exploited to access new molecular diversity. Coinfiltration of
A. tumefaciens strains each containing different expression constructs provides a powerful means of rapidly expressing novel enzyme combinations, including enzymes from different plant species in a process frequently termed combinatorial biosynthesis. This type of approach has recently been demonstrated for accessing new diterpene scaffolds, which may be formed from GGDP in a two-step process performed by the combined action of discrete class II and class I cyclases (Zerbe and Bohlmann 2015). By employing systematic transient expression of combinations of 11 class II and 9 class I cyclases, Andersen-Ranberg et al. (2016) successfully obtained 51 functional pairings, resulting in a significant proportion of "new-to-nature" products with potential utility as new molecular scaffolds (Fig. 3a).

Combinatorial biosynthesis has also been exploited for diversification of terpene scaffolds. Much of the terpene structural diversity present in nature can be attributed to the action of tailoring enzymes, such as cytochrome P450s, glycosyl- and acyltransferases, which can produce thousands of variants from commonly occurring scaffolds (Hamberger and Bak 2013; Seki et al. 2015). The ubiquitous triterpene $\beta$-amyrin is one such example and derivatives of this molecule from different plants have a remarkable array of properties as sweeteners, anti-inflammatory-, anti-fungal- and adjuvant agents. However, an understanding of the importance of individual structural modifications with respect to activity are poorly understood, and the scaffolds are difficult to selectively modify using current chemical synthetic chemistry methods. Cytochrome P450s are capable of performing highly regio- and stereoselective oxidation of their substrates and hence provide an excellent resource for achieving structural diversification. As a proof of concept, transient coexpression of a $\beta$-amyrin synthase with individual $\beta$-amyrinoxidising P450s and combinations of pairs of P450s was performed, resulting in production of a series of $\beta$-amyrin derivatives featuring the specific modifications introduced by the respective P450s (Fig. 3b) (Reed et al. 2017). Several of these products were not represented in natural product databases. A number of these products were purified from $N$. benthamiana in up to gram quantities allowing for the biological activity of the products to be explored. The ability to selectively oxidise the scaffolds in this manner could provide functional handles for further modification using other biosynthetic enzymes, or semisynthetic chemistry. The ability to coinfiltrate multiple $A$. tumefaciens strains allows the same vectors and selectable markers to be used for each gene, irrespective of how they will be ultimately expressed in planta. Performing this procedure is relatively simple and can be adopted by a researcher with relatively little molecular biology training.

Ultimately, the approaches above could be expanded to large-scale assembly and screening of combinatorial libraries for new molecules. However, at present, this approach 

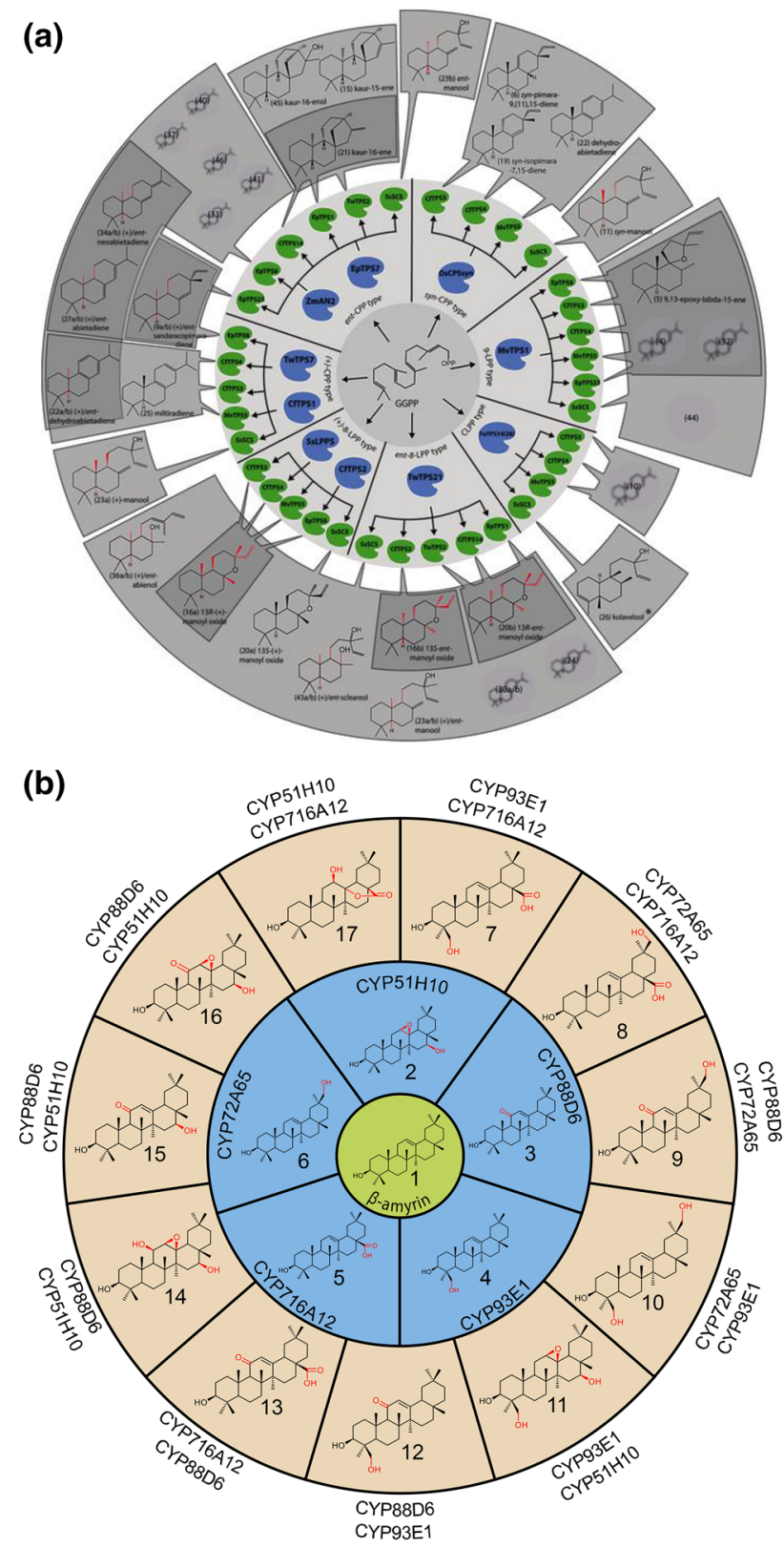

Fig. 3 Combinatorial biosynthesis in $N$. benthamiana. a Production of diterpene scaffolds in $N$. benthamiana though combination of pairs of class II (inner circle, blue) and class I (inner circle, green) cyclases. Bonds in a specified stereochemical configuration formed through stereoselective controlled synthesis are highlighted in red. Bonds in a specified stereochemical configuration for which there is no biosynthetic access to the other configuration are shown in black. The $\mathbf{a}$ and $\mathbf{b}$ suffixes indicate (+) and ent configurations, respectively, as dictated by the class II diterpene synthases. Diterpenes with unknown structure are shaded in grey. *Clerodane diterpenes. b Production of oxygenated triterpenes. The products of individual P450s acting upon $\beta$-amyrin (centre) are shown in the inner blue circle. The major combinatorial products arising from pairs of P450s are shown in the outer circle. a adapted from Andersen-Ranberg et al. (2016), b adapted from Reed et al. (2017) is limited by the relatively small number of characterised genes, representing only a tiny fraction of the thousands of terpene biosynthetic pathways in nature. Discovery of new biosynthetic pathways will be facilitated by the increasing availability of sequence data from non-model and noncrop species thanks to projects such as the one thousand plants (1KP) database (Matasci et al. 2014), its successor ten thousand plants (10KP) project (Normile 2017), and the JGI Open Green Genomes initiative (https://jgi.doe.gov/ csp-2018-leebens-mack-open-green-genomes-initiative/). Nevertheless, even the genomes of well-characterised model species such as A. thaliana encode a significant wealth of biosynthetic enzymes and pathways which are uncharacterised due to the products being present either at trace levels or under specialised conditions. It is now recognised that a number of diverse plant specialised metabolic pathways are arranged into 'operon-like' clusters, whereby the enzymes required for a biochemical pathway are encoded by a series of physically colocalised genes (Nutzmann et al. 2016). This provides an opportunity to discover novel biosynthetic pathways by developing algorithms to mine for the presence of such clusters (Medema and Osbourn 2016; Owen et al. 2017). Once identified, expression of these colocalised genes in $N$. benthamiana can be performed to determine their functional relevance. This approach has previously been applied for identifying terpenoid biosynthetic pathways from species including from A. thaliana, cucumber and Lotus japonicus (Boutanaev et al. 2015; Huang et al. 2017b; Krokida et al. 2013). Future work in this area will also benefit from the rapidly reducing cost of gene synthesis, which could ultimately lead to the ability to screen entire libraries of candidate genes for activity (Kosuri and Church 2014).

\section{Issues for the future}

It is clear that $N$. benthamiana shows a great deal of promise for the production of terpenes and there are a growing number of examples, where this host has been used to obtain isolated yields of the target compounds in milligram to gram quantities (Tables 1, 2) (Andersen-Ranberg et al. 2016; Huang et al. 2017a, b; Reed et al. 2017). At the same time, one of the drawbacks to achieving this level of production is the matter of scale. For example, while infiltration of a single leaf by hand (Fig. 1a/b) is sufficient for most analytical work, translating this approach to infiltrate the volume of plants required for milligram-to-gram quantities of the target compound is laborious. To address this challenge, we and others have utilised vacuum infiltration of $N$. benthamiana (Andersen-Ranberg et al. 2016; Huang et al. 2017a, b; Reed et al. 2017). This process involves inverting the aerial parts of $N$. benthamiana plants into a bath of A. tumefaciens and using a vacuum to draw the air from the interstitial spaces 
between the leaf cells (Reed et al. 2017). Upon equilibration to atmospheric pressure, the bacterial suspension is drawn into the leaves. This process allows for simultaneous batchwise infiltration of multiple plants at once. This process has been applied on a commercial scale for the production of pharmaceutical proteins in $N$. benthamiana (Holtz et al. 2015). For the academic lab, such devices can be constructed relatively cheaply with commercially available parts.

One of the issues that has confounded production of some molecules has been the observation of unintended side products resulting from modification of the target compound by endogenous enzymes, resulting in oxidation, glycosylation or dephosphorylation (Brückner and Tissier 2013; Dong et al. 2016; Khakimov et al. 2015; Liu et al. 2011; Wang et al. 2016). Although such reactions may give rise to new bioactive molecules (Liu et al. 2014), such crosstalk with the host metabolism is generally undesirable. Genome editing approaches (Li et al. 2013; Nekrasov et al. 2013) might, therefore, be employed to limit this phenomenon by knocking out endogenous sugar transferases or oxidases which crosstalk with the heterologously expressed pathway. Alternatively, approaches to minimise the potential for crosstalk with endogenous enzymes may be utilised to circumvent this problem. For example, transient expression of the Lipid Transfer Protein 3 (LTP3) and Pleiotropic Drug Resistance Transporter 2 (PD2) from Artemisia annua in N. benthamiana resulted in increased transport of artemisinin precursors to the apoplast, thereby reducing loss of intermediates to glycosylation by cytosolic endogenous glycosyltransferases (Wang et al. 2016).

Although the ability to infiltrate multiple strains offers a rapid way to assemble new gene combinations, the density of the bacterial inoculum must be increased with coinfiltration of additional A. tumefaciens cultures, which imposes practical limitations on the maximum number of different constructs that can be coexpressed (Montague et al. 2011). Therefore, integration of multiple genes into a single T-DNA provides a solution to this problem. One possible approach to this is to fuse multiple genes into a single open reading frame, separated by a viral peptide signal (2A) which results in ribosomal skipping and production of the individual proteins (van Herpen et al. 2010). An additional drawback of coinfiltration is the lack of fine control over the stoichiometry of the various coexpressed proteins due to differences in the efficiency of T-DNA delivery from different $A$. tumefaciens strains to individual leaf cells (Montague et al. 2011). Control over protein stoichiometry during transient expression has previously been demonstrated by modulating translation efficiency of the expressed genes in a single T-DNA with different 5' UTR leader sequences. This approach allowed optimised production of Bluetongue virus VLPs in N. benthamiana (Thuenemann et al. 2013). For metabolic engineering, such an approach might be applied for controlling the stoichiometry of subunits of heteromeric enzymes, balancing enzymes and their respective coenzymes (such as cytochrome P450s and reductases) or optimising multiple steps in a pathway to balance flux through a biosynthetic pathway. Similarly, this could be achieved through use of synthetic promoters designed to have various strengths and which are controlled by a single orthogonal transcription factor. This approach has been demonstrated for engineering diterpene production levels upon transient expression in $N$. benthamiana (Brückner et al. 2015). Modular cloning techniques such as the type II restriction enzyme-based Golden Gate system (Weber et al. 2011) enable the researcher to choose from a library of validated parts including promoters, terminators and UTRs, thereby allowing fine tuning over expression of individual proteins (Engler et al. 2014).

\section{Conclusions and perspectives}

Utilising heterologous host production systems has immense potential for accessing the thousands of bioactive plant specialised metabolites present in nature. Microbial production systems have demonstrated the potential of this approach to meet demand for high-value plant chemicals. The successful engineering of Saccharomyces cerevisiae for production of artemisinin serves as a flagship example (Paddon and Keasling 2014; Paddon et al. 2013). Other recent examples have also emerged of engineering this host to produce other complex high-value alkaloids (Brown et al. 2015; Galanie et al. 2015; Li and Smolke 2016). Nevertheless, these efforts have required extensive optimisation of the genetic background of the host strain in addition to expression of the biosynthetic pathway of interest to subvert yeast metabolism towards classes of products it has not evolved to produce. Furthermore, the yields of the end products are still in the microgram per litre range in many cases, thus highlighting the need to continue to explore other host systems. The numbers of studies demonstrating the purification of compounds derived from transient expression in plants have increased in recent years, reflecting the increasing popularity of this system (Andersen-Ranberg et al. 2016; Dickschat et al. 2017; Geisler et al. 2013; Huang et al. 2017a, b; Reed et al. 2017). As described above, transient expression can be achieved at industrial scale, and is currently used for production of pharmaceutical proteins by various companies (Sack et al. 2015). The linear nature of scaling vacuum infiltration is important for translating small scale experiments from an academic lab to commercial setting. This scalability, coupled with the rapid turnaround time of transient expression, allows vaccines to be produced within a matter of weeks and mobilised in response to emerging epidemics (Marsian and Lomonossof 2016; Owen et al. 2017). This principle could similarly be applied for on-demand access to small molecules. With 
this in mind, transient plant expression has the potential to be a disruptive technology for metabolic engineering of plant bioactives and other high-value chemicals.

Author contribution statement JR and AO co-wrote the manuscript.

\begin{abstract}
Acknowledgements This work was supported by a National Institutes of Health Genome to Natural Products Network award U101GM110699 (A.O., J.R.). A.O.'s laboratory is funded by the UK Biotechnological and Biological Sciences Research Council (BBSRC) Institute Strategic Programme Grant "Molecules from Nature" (BB/P012523/1) and BBSRC-funded OpenPlant Synthetic Biology Research Centre grant (BB/L014130/1) and the John Innes Foundation. We would like to thank Andrew Davis and Thomas Louveau for photography and Aymeric Leveau/NRP image library for the picture of the infiltrated leaf in Fig. 1b.
\end{abstract}

\section{Compliance with ethical standards}

Conflict of interest The authors declare no conflict of interest.

Open Access This article is distributed under the terms of the Creative Commons Attribution 4.0 International License (http://creativeco mmons.org/licenses/by/4.0/), which permits unrestricted use, distribution, and reproduction in any medium, provided you give appropriate credit to the original author(s) and the source, provide a link to the Creative Commons license, and indicate if changes were made.

\section{References}

Andersen-Ranberg J et al (2016) Expanding the landscape of diterpene structural diversity through stereochemically controlled combinatorial biosynthesis. Angew Chem 55:2142-2146. https://doi. org/10.1002/anie.201510650

Andersen-Ranberg J et al (2017) Synthesis of C-glucosylated octaketide anthraquinones in Nicotiana benthamiana by using a multispecies-based biosynthetic pathway. ChemBioChem 18:18931897. https://doi.org/10.1002/cbic.201700331

Atanasov AG et al (2015) Discovery and resupply of pharmacologically active plant-derived natural products: a review. Biotechnol Adv 33:1582-1614. https://doi.org/10.1016/j.biotechadv.2015.08.001

Bick JA, Lange BM (2003) Metabolic cross talk between cytosolic and plastidial pathways of isoprenoid biosynthesis: unidirectional transport of intermediates across the chloroplast envelope membrane. Arch Biochem Biophys 415:146-154

Boutanaev AM, Moses T, Zi J, Nelson DR, Mugford ST, Peters RJ, Osbourn A (2015) Investigation of terpene diversification across multiple sequenced plant genomes. Proc Natl Acad Sci USA 112:E81-88. https://doi.org/10.1073/pnas.1419547112

Brown S, Clastre M, Courdavault V, O'Connor SE (2015) De novo production of the plant-derived alkaloid strictosidine in yeast. Proc Natl Acad Sci USA 112:3205-3210. https://doi.org/10.1073/ pnas. 1423555112

Brückner K, Tissier A (2013) High-level diterpene production by transient expression in Nicotiana benthamiana. Plant Methods 9:46. https://doi.org/10.1186/1746-4811-9-46

Brückner K, Schafer P, Weber E, Grutzner R, Marillonnet S, Tissier A (2015) A library of synthetic transcription activator-like effector-activated promoters for coordinated orthogonal gene expression in plants. Plant J 82:707-716. https://doi.org/10.1111/ tpj. 12843

Cankar K et al (2015) (+)-Valencene production in Nicotiana benthamiana is increased by down-regulation of competing pathways. Biotechnol J 10:180-189. https://doi.org/10.1002/biot.201400288

Crocoll C, Mirza N, Reichelt M, Gershenzon J, Halkier BA (2016) Optimization of engineered production of the glucoraphanin precursor dihomomethionine in Nicotiana benthamiana. Front Bioeng Biotechnol 4:14

Dickschat JS, Rinkel J, Rabe P, Beyraghdar Kashkooli A, Bouwmeester HJ (2017) 18-Hydroxydolabella-3,7-diene synthase-a diterpene synthase from Chitinophaga pinensis. Beilstein J Org Chem 13:1770-1780. https://doi.org/10.3762/bjoc.13.171

Disch A, Hemmerlin A, Bach TJ, Rohmer M (1998) Mevalonatederived isopentenyl diphosphate is the biosynthetic precursor of ubiquinone prenyl side chain in tobacco BY-2 cells. Biochem J 331(Pt 2):615-621

Dong L, Jongedijk E, Bouwmeester H, Van Der Krol A (2016) Monoterpene biosynthesis potential of plant subcellular compartments. New Phytol 209:679-690. https://doi.org/10.1111/ nph.13629

Eljounaidi K et al (2014) Cytochrome P450s from Cynara cardunculus L. CYP71AV9 and CYP71BL5, catalyze distinct hydroxylations in the sesquiterpene lactone biosynthetic pathway. Plant Sci 223:59-68. https://doi.org/10.1016/j.plantsci.2014.03.007

Engler C et al (2014) A golden gate modular cloning toolbox for plants. ACS Synth Biol 3:839-843. https://doi.org/10.1021/sb4001504

Farhi M et al (2011) Generation of the potent anti-malarial drug artemisinin in tobacco. Nat Biotechnol 29:1072-1074. https://doi. org/10.1038/nbt.2054

Fischer MJ et al (2013) Specificity of Ocimum basilicum geraniol synthase modified by its expression in different heterologous systems. J Biotechnol 163:24-29. https://doi.org/10.1016/j.jbiot ec.2012.10.012

Galanie S, Thodey K, Trenchard IJ, Filsinger Interrante M, Smolke CD (2015) Complete biosynthesis of opioids in yeast. Science 349:1095-1100. https://doi.org/10.1126/science.aac9373

Geisler K et al (2013) Biochemical analysis of a multifunctional cytochrome P450 (CYP51) enzyme required for synthesis of antimicrobial triterpenes in plants. Proc Natl Acad Sci USA 110:E3360-3367. https://doi.org/10.1073/pnas.1309157110

Geu-Flores F, Nielsen MT, Nafisi M, Moldrup ME, Olsen CE, Motawia MS, Halkier BA (2009) Glucosinolate engineering identifies a gamma-glutamyl peptidase. Nat Chem Biol 5:575-577. https:// doi.org/10.1038/nchembio.185

Gnanasekaran T, Vavitsas K, Andersen-Ranberg J, Nielsen AZ, Olsen CE, Hamberger B, Jensen PE (2015) Heterologous expression of the isopimaric acid pathway in Nicotiana benthamiana and the effect of $\mathrm{N}$-terminal modifications of the involved cytochrome P450 enzyme. J Biol Eng 9:24. https://doi.org/10.1186/s1303 6-015-0022-Z

Hamberger B, Bak S (2013) Plant P450s as versatile drivers for evolution of species-specific chemical diversity. Philos Trans R Soc B 368:20120426. https://doi.org/10.1098/rstb.2012.0426

Hasan MM et al (2014) Metabolic engineering of Nicotiana benthamiana for the increased production of taxadiene. Plant Cell Rep 33:895-904. https://doi.org/10.1007/s00299-014-1568-9

Hemmerlin A et al (2003) Cross-talk between the cytosolic mevalonate and the plastidial methylerythritol phosphate pathways in tobacco bright yellow-2 cells. J Biol Chem 278:26666-26676. https://doi. org/10.1074/jbc.M302526200

Hemmerlin A, Harwood JL, Bach TJ (2012) A raison d'etre for two distinct pathways in the early steps of plant isoprenoid biosynthesis? Prog Lipid Res 51:95-148. https://doi.org/10.1016/j.plipr es.2011.12.001 
Holtz BR et al (2015) Commercial-scale biotherapeutics manufacturing facility for plant-made pharmaceuticals. Plant Biotechnol J 13:1180-1190. https://doi.org/10.1111/pbi.12469

Huang AC, Hong YJ, Bond AD, Tantillo DJ, Osbourn A (2017a) Diverged plant terpene synthases reroute the carbocation cyclization path towards the formation of unprecedented $6 / 11 / 5$ and 6/6/7/5 sesterterpene scaffolds. Angew Chem 57:5. https://doi. org/10.1002/anie.201711444

Huang AC, Kautsar SA, Hong YJ, Medema MH, Bond AD, Tantillo DJ, Osbourn A (2017b) Unearthing a sesterterpene biosynthetic repertoire in the Brassicaceae through genome mining reveals convergent evolution. Proc Natl Acad Sci. https://doi. org/10.1073/pnas.1705567114

Khakimov B et al (2015) Identification and genome organization of saponin pathway genes from a wild crucifer, and their use for transient production of saponins in Nicotiana benthamiana. Plant J 84:478-490. https://doi.org/10.1111/tpj.13012

Kosuri S, Church GM (2014) Large-scale de novo DNA synthesis: technologies and applications. Nat Methods 11:499. https://doi. org/10.1038/nmeth.2918

Krokida A et al (2013) A metabolic gene cluster in Lotus japonicus discloses novel enzyme functions and products in triterpene biosynthesis. New Phytol 200:675-690. https://doi.org/10.1111/ nph. 12414

Lau W, Sattely ES (2015) Six enzymes from mayapple that complete the biosynthetic pathway to the etoposide aglycone. Science 349:1224-1228. https://doi.org/10.1126/science.aac7202

Li Y, Smolke CD (2016) Engineering biosynthesis of the anticancer alkaloid noscapine in yeast. Nat Commun 7:12137. https://doi. org/10.1038/ncomms12137

Li J-F et al (2013) Multiplex and homologous recombination-mediated genome editing in Arabidopsis and Nicotiana benthamiana using guide RNA and Cas9. Nat Biotechnol 31:688. https://doi. org/10.1038/nbt.2654

Lichtenthaler HK, Schwender J, Disch A, Rohmer M (1997) Biosynthesis of isoprenoids in higher plant chloroplasts proceeds via a mevalonate-independent pathway. FEBS Lett 400:271-274

Liu Q et al (2011) Reconstitution of the costunolide biosynthetic pathway in yeast and Nicotiana benthamiana. Plos One 6:e23255. https://doi.org/10.1371/journal.pone.0023255

Liu Q et al (2014) Elucidation and in planta reconstitution of the parthenolide biosynthetic pathway. Metab Eng 23:145-153. https ://doi.org/10.1016/j.ymben.2014.03.005

Marsian J, Lomonossoff GP (2016) Molecular pharming-VLPs made in plants. Curr Opin Biotechnol 37:201-206. https://doi. org/10.1016/j.copbio.2015.12.007

Matasci N et al (2014) Data access for the 1000 Plants (1KP) project. Gigascience 3:17. https://doi.org/10.1186/2047-217X-3-17

Medema MH, Osbourn A (2016) Computational genomic identification and functional reconstitution of plant natural product biosynthetic pathways. Nat Prod Rep 33:951-962. https://doi. org/10.1039/c6np00035e

Miettinen K et al (2014) The seco-iridoid pathway from Catharanthus roseus. Nat Commun 5:3606. https://doi.org/10.1038/ ncomms 4606

Montague NP, Thuenemann EC, Saxena P, Saunders K, Lenzi P, Lomonossoff GP (2011) Recent advances of cowpea mosaic virus-based particle technology. Hum Vaccines 7:383-390

Nekrasov V, Staskawicz B, Weigel D, Jones JDG, Kamoun S (2013) Targeted mutagenesis in the model plant Nicotiana benthamiana using Cas9 RNA-guided endonuclease. Nat Biotechol 31:691693. https://doi.org/10.1038/nbt.2655

Normile D (2017) Plant scientists plan massive effort to sequence 10,000 genomes. http://www.sciencemag.org/news/2017/07/ plant-scientists-plan-massive-effort-sequence-10000-genom es. Accessed 26 Feb 2018
Nutzmann HW, Huang A, Osbourn A (2016) Plant metabolic clusters-from genetics to genomics. New Phytol 211:771-789. https://doi.org/10.1111/nph.13981

Owen C, Patron NJ, Huang A, Osbourn A (2017) Harnessing plant metabolic diversity. Curr Opin Chem Biol 40:24-30. https://doi. org/10.1016/j.cbpa.2017.04.015

Paddon CJ, Keasling JD (2014) Semi-synthetic artemisinin: a model for the use of synthetic biology in pharmaceutical development. Nat Rev Microbiol 12:355-367. https://doi.org/10.1038/nrmicro3240

Paddon CJ et al (2013) High-level semi-synthetic production of the potent antimalarial artemisinin. Nature 496:528-532. https:// doi.org/10.1038/nature12051

Pfalz M, Mikkelsen MD, Bednarek P, Olsen CE, Halkier BA, Kroymann J (2011) Metabolic engineering in Nicotiana benthamiana reveals key enzyme functions in Arabidopsis indole glucosinolate modification. Plant Cell 23:716-729. https://doi. org/10.1105/tpc. 110.081711

Polakowski T, Stahl U, Lang C (1998) Overexpression of a cytosolic hydroxymethylglutaryl-CoA reductase leads to squalene accumulation in yeast. Appl Microbiol Biotechnol 49:66-71

Polturak G et al (2016) Elucidation of the first committed step in betalain biosynthesis enables the heterologous engineering of betalain pigments in plants. New Phytol 210:269-283. https:// doi.org/10.1111/nph.13796

Rajniak J, Barco B, Clay NK, Sattely ES (2015) A new cyanogenic metabolite in Arabidopsis required for inducible pathogen defence. Nature 525:376-379. https://doi.org/10.1038/natur e14907

Reed J et al (2017) A translational synthetic biology platform for rapid access to gram-scale quantities of novel drug-like molecules. Metab Eng 42:185-193. https://doi.org/10.1016/j.ymben .2017 .06 .012

Sack M, Hofbauer A, Fischer R, Stoger E (2015) The increasing value of plant-made proteins. Curr Opin Biotechnol 32:163170. https://doi.org/10.1016/j.copbio.2014.12.008

Schuhr CA et al (2003) Quantitative assessment of crosstalk between the two isoprenoid biosynthesis pathways in plants by NMR spectroscopy. Phytochem Rev 2:3-16. https://doi.org/10.1023/ b:Phyt.0000004180.25066.62

Seki H, Tamura K, Muranaka T (2015) P450s and UGTs: Key players in the structural diversity of triterpenoid saponins. Plant Cell Physiol 56:1463-1471. https://doi.org/10.1093/pcp/pcv062

Thuenemann EC, Meyers AE, Verwey J, Rybicki EP, Lomonossoff GP (2013) A method for rapid production of heteromultimeric protein complexes in plants: assembly of protective bluetongue virus-like particles. Plant Biotechnol J 11:839-846. https://doi. org/10.1111/pbi.12076

Ting HM, Delatte TL, Kolkman P, Misas-Villamil JC, van der Hoorn RA, Bouwmeester HJ, van der Krol AR (2015) SNARE-RNAi results in higher terpene emission from ectopically expressed caryophyllene synthase in Nicotiana benthamiana. Mol Plant. https://doi.org/10.1016/j.molp.2015.01.006

van Herpen TW, Cankar K, Nogueira M, Bosch D, Bouwmeester HJ, Beekwilder J (2010) Nicotiana benthamiana as a production platform for artemisinin precursors. Plos One 5:e14222. https ://doi.org/10.1371/journal.pone.0014222

Vasilev N et al (2014) Comparison of plant-based expression platforms for the heterologous production of geraniol. Plant Cell Tissue Organ Cult 117:373-380. https://doi.org/10.1007/s1124 0-014-0446-z

Vranova E, Coman D, Gruissem W (2013) Network analysis of the MVA and MEP pathways for isoprenoid synthesis. Annu Rev Plant Biol 64:665-700. https://doi.org/10.1146/annurev-arpla nt-050312-120116

Wang B et al (2016) Transient production of artemisinin in Nicotiana benthamiana is boosted by a specific lipid transfer protein from 
A. апnиа. Metab Eng 38:159-169. https://doi.org/10.1016/j. ymben.2016.07.004

Weber E, Engler C, Gruetzner R, Werner S, Marillonnet S (2011) A modular cloning system for standardized assembly of multigene constructs. Plos One 6:e16765. https://doi.org/10.1371/journ al.pone. 0016765

Wu S, Schalk M, Clark A, Miles RB, Coates R, Chappell J (2006) Redirection of cytosolic or plastidic isoprenoid precursors elevates terpene production in plants. Nat Biotechnol 24:1441-1447. https ://doi.org/10.1038/nbt1251
Wu S, Jiang Z, Kempinski C, Eric Nybo S, Husodo S, Williams R, Chappell J (2012) Engineering triterpene metabolism in tobacco. Planta 236:867-877. https://doi.org/10.1007/s00425-012-1680-4

Yin JL, Wong WS, Jang IC, Chua NH (2017) Co-expression of peppermint geranyl diphosphate synthase small subunit enhances monoterpene production in transgenic tobacco plants. New Phytol 213:1133-1144. https://doi.org/10.1111/nph.14280

Zerbe P, Bohlmann J (2015) Plant diterpene synthases: exploring modularity and metabolic diversity for bioengineering. Trends Biotechnol 33:419-428. https://doi.org/10.1016/j.tibtech.2015.04.006 